\title{
Management of cataract surgery in a high myope
}

\author{
Helen Seward, Richard Packard, David Allen
}

\begin{abstract}
Management problem
A 52 year old high myope presents with misty vision as a result of cataracts. With his contact lenses he sees $6 / 9$ part right eye, 6/9 left eye and reads $\mathrm{N} 8$ right eye, N6 left eye with glasses.

He is wearing $-12.00 /-2.00 \times 75$ right eye, $-11.00 /-1.00 \times 90$ left eye. He has bilateral posterior subcapsular plaques $\mathbf{R}>\mathbf{L}$ and myopic retinal degeneration. Axial lengths measured using SRK-T are $30.60 \mathrm{~mm}$ right eye, $30.31 \mathrm{~mm}$ left eye.
\end{abstract}

He is a keen runner and would like good unaided distance vision postsurgery. He is very light sensitive and finds tonometry difficult.

How would you manage this patient's cataract surgery, in particular the type of anaesthetic, the surgical management plan, and the choice of intraocular lens?

\section{View 1}

\section{Richard Packard}

This patient, who is highly myopic presents with reasonably good distance vision but compromised reading. However, we may assume that his distance VA in glare conditions will be much worse because of his posterior subcapsular plaques and that it is from this that his problems really arise. He has also expressed a desire for good unaided distance vision postsurgery which will require some form of correction for his astigmatism.

\section{Preoperative assessment}

As part of the preoperative assessment the following tests should be carried out to assist in developing the appropriate surgical plan.

(1) Full fundal examination has shown myopic degeneration. Assess these lesions in the retina as they may require treatment either before or immediately after the surgery.

(2) Ultrasonic A-scan using the appropriate

Windsor Hospital, Surrey, UK R Packard consultant ophthalmologist formula and A-constant. As used here SRK-T is accurate for high myopes. As an alternative the Holladay II may be used, which requires the white to white corneal measurement. When doing the A scan be aware that high myopes may have posterior polar anomalies such as posterior staphylomata which can cause inaccurate biometry results if the patient's ability to fix is in any way impaired. Also the fovea may not be on the visual axis and there may be a wide angle kappa which can lead to further inaccuracies.

(3) An ultrasonic B scan should be done to establish if any staphylomata are present and if need be the axial length can also be confirmed using this. It is also useful to measure the anterior chamber depth as this may help to make the IOL calculation more accurate.

(4) If the cataract is not too dense measurement by optical means using a Zeiss IOL master can provide a more accurate result where irregularities at the posterior of the eye are a possibility.

(5) Corneal topography will be required to assess the appropriate meridian, type, and amount of astigmatic correction.

\section{Informed consent}

As to the informed consent in relation to anaesthetic and surgery, the patient will need full explanation of the risks of surgery as well as the possibilities of achieving the desired visual result. Recent studies ${ }^{1}{ }^{2}$ indicate that the risk of retinal detachment is much less than with earlier techniques. Although modern measuring techniques are very good, at this axial length the patient must be warned that plus or minus 2 dioptres from desired outcome may occur. One study $^{3}$ has shown that $69 \%$ of highly myopic patients were within 1 dioptre of desired refractive outcome. In this situation erring on the side of slight myopia of about -0.75 dioptres is probably a safe option. Some myopes wish to retain the ability to see near to without glasses and in this instance aim for -2.5 dioptres as an end point. ${ }^{4}$

\section{Anaesthesia}

We are told that this patient is very photophobic and sensitive even to tonometry. Topical anaesthesia is not ideal because of these two reasons. We are also told that the axial length is greater than $30 \mathrm{~mm}$. In this situation retrobulbar and peribulbar anaesthesia are not desirable options because of the risk of globe penetration. ${ }^{5}$ Although sub-Tenon's injection could be used there is still the problem of blepharospasm associated with the patient's photophobia. On balance, as he is fit general anaesthesia with one of the modern gaseous 
agents, such as sevofluorane, or total intravenous anaesthesia, using propofol in conjunction with a laryngeal mask, is my preferred option. We normally supplement the anaesthesia with benoxinate $0.4 \%$ drops. Patients wake very rapidly at the end of the operation without coughing and the technique works very well for day case surgery.

\section{Bilateral surgery?}

If the patient is to have general anaesthesia it is worth considering bilateral simultaneous surgery. ${ }^{6-8}$ The advantage for the patient is only one anaesthetic and a minimal period of visual disruption caused by the surgery. If only one eye is done, particularly in patients who wear glasses (and this is, therefore, less of an issue in this particular man), the aneisokonia in high myopes is profound. The theoretical risks of problems with bilateral surgery have never been fully worked out and this author has used bilateral surgery for some years without difficulties. It is particularly useful for patients with significant refractive errors, those who are educationally subnormal, and patients coming to the United Kingdom from overseas. Providing that the surgery is treated as two separate operations, with full change of everything, the risks of infection should be no greater than doing the eyes apart. The only reason for not taking this approach is if there are doubts about the accuracy of the biometry and if it is felt to be important to see the outcome for the first eye before deciding on an IOL power for the second eye. In any event both eyes should be done in close temporal proximity for the patient's visual comfort, even if the second eye has only minimal cataract.

\section{Aspects of phacoemulsification in high myopes}

The type and position of the incisions need to be considered. If we confine ourselves to the right eye initially we see that there is astigmatism of -2.00 dioptres at 75 degrees. If we assume that this all on the cornea and is borne out by topography how are we to reduce it? The meridian of the high $\mathrm{K}$ value will be 165 degrees, a $3.5 \mathrm{~mm}$ clear corneal phaco incision in this position will produce between 0.5 and 0.75 dioptres of cylinder reduction. To achieve the other 1.5 dioptres of change a limbal relaxing incision of 1.5 clock hours at 180 degrees from the phaco wound at a depth of $600 \mu \mathrm{m}$ is required (Fig 1). Other approaches may be used ${ }^{10}$ but this is my current approach. These incisions are made before the removal of the cataract.

As the anterior chamber will be abnormally deep do not make the phaco tunnel too long as this will cause distortion in the cornea as instruments are manoeuvred in the tunnel.

Myopes normally dilate extremely well. Care must be taken when making the capsulorhexis to ensure that it is not too large, as the overall diameter of the anterior chamber is greater too. Ideally, as the edge of the IOL optic should be covered by capsule the ideal size is between 5 and $5.5 \mathrm{~mm}$. The capsule in highly myopic

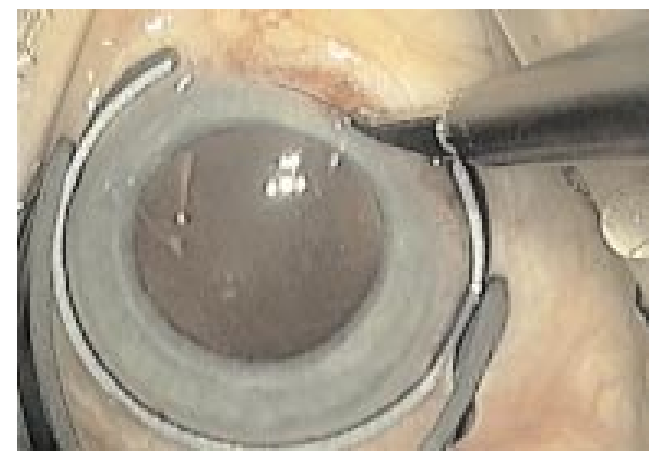

Figure 1 Limbal relaxing incision for astigmatism correction.

patients aged 52 years can be surprisingly elastic with a tendency to tear peripherally. There is a temptation not to deepen the anterior chamber too much because it makes the capsulorhexis more difficult; it is safer to have the capsule flattened with viscoelastic.

We are told that this man's cataract is largely posterior subcapsular. When the hydrodissection is carried out in a patient of this age with a cataract of this type the nucleus may start to prolapse from the capsular bag owing to its softness. This is to your advantage as it will mean that much of the nucleus and epinucleus can be removed without the phaco probe being too vertical.

If the nucleus is likely to behave as above, I would use my high vacuum setting. This way most, if not all, of the nucleus will be able to be removed with vacuum alone. There may be the need to use low phaco power simply to massage the lens material through the phaco port. There should be good "followability" of nuclear material as it has been thoroughly freed by the hydrodissection.

If the nucleus remains in the capsular bag it is important that hydrodissection is sufficient to free the lens matter totally. I would also recommend carrying out hydrodelineation of what will probably be a relatively small nucleus from the epinucleus. It is important to know the extent of the nucleus when one has the phaco probe at a much steeper angle than usual so that the epinucleus and then the capsule are not penetrated inadvertently.

When the phaco probe is first placed in the eye and irrigation engaged the anterior chamber can deepen alarmingly (Fig 2). This can make any sculpting manoeuvres much more difficult because of the near vertical position of the phaco probe. Lower the bottle to allow the chamber to become shallower and also place the second instrument in the side port to permit some leakage.

After the nucleus has been removed there may be extensive epinucleus present. If adequate hydrodissection has not been achieved great care must be taken to mobilise this. Not to do so may lead to both capsule and epinucleus being engaged in the I/A tip at the same time without the surgeon's knowledge, with unfortunate results. Viscodissection at the edge of the capsulorhexis will normally lift the epinucleus away from the posterior capsule to allow for its easier removal. 


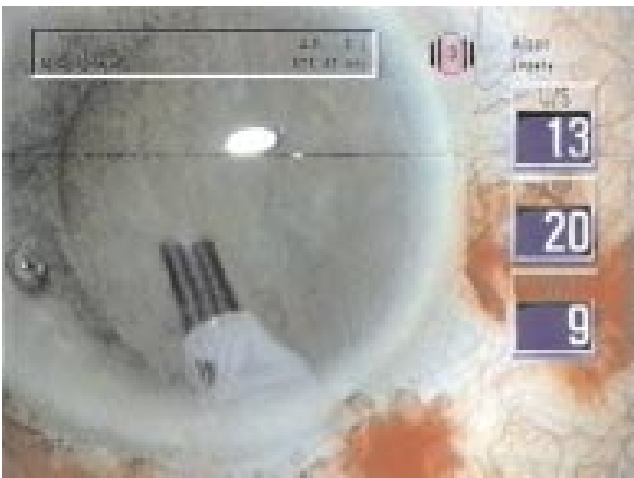

Figure 2 Abnormally deep anterior chamber.

We are told that this patient has posterior subcapsular plaques; these may remain after the I/A and will need to be removed. There are several approaches to this:

(1) Attempt to polish the plaque from the capsule. A number of different devices are available for this. I turn the irrigation probe of the bimanual I/A sideways and use the port to scrub the capsule.

(2) Fill the eye with viscoelastic and then try to lift the edge of the plaque with a needle and then strip it off with capsulorhexis forceps. This is easier than it sounds but if the capsule tears convert to posterior capsulorhexis.

(3) Set out to do a posterior capsulorhexis (Fig 3). There has been a report on clear capsules with posterior capsulorhexis, where the incidence of retinal detachment is acceptable ${ }^{11}$; this technique is gentler than YAG laser capsulotomy with no shock wave to the posterior pole.

(4) If the plaque on the posterior capsule is not too thick leave it alone and see what is achieved as the best postoperative vision.

\section{Choosing the IOL}

As this patient has an axial length of over 30 $\mathrm{mm}$ he will almost certainly require an IOL power in the range -3 to +3 . Until recently foldable IOLs in this range were very limited and most surgeons would have had to opt for a PMMA rigid lens. These lenses usually had a diameter of 6-7 $\mathrm{mm}$ and therefore many of the aspects of the small incision were lost. There was also the issue of posterior capsular opacity with lenses of this type as it would be nice to avoid a capsulotomy if possible. Recently, a range of lenses from +5 to -5 has been produced, in the Alcon Acrysof range, the MA60MA. This lens has an optic diameter of 6

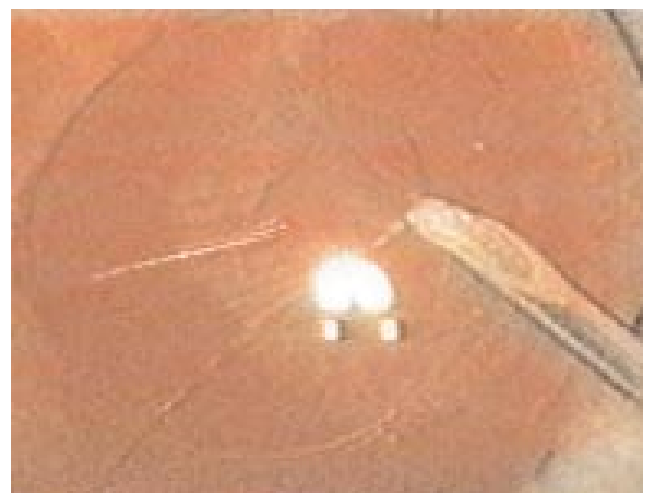

Figure 3 Starting a posterior capsulorhexis in the presence of a capsular plaque.

$\mathrm{mm}$, it is meniscus shaped with the convex surface against the posterior capsule. A matt edge should minimise the possibility of edge glare. IOLs with sharp edges, as here, have been shown to reduce considerably the risk of posterior capsule opacification (PCO), ${ }^{12-14}$ and this, as well as the chance to implant through a 3.5 $\mathrm{mm}$ self sealing wound, are added advantages.

1 Alldredge CD, Elkins B, Alldredge OC. Retinal detachment following phacoemulsification in highly myopic cataract following phacoemulsification in highly myopic

2 Jacobi FK, Hessemer VJ. Pseudophakic retinal detachment in high axial myopia. f Cataract Refract Surg 1997;23:1095102 .

3 Kohnen S, Brauweiler PJ. First results of cataract surgery and implantation of negative power intraocular lenses in highly myopic eyes. F Cataract Refract Surg 1996;22:41620.

4 Kora Y, Yaguchi S, Inatomi M, et al. Preferred postoperative refraction after cataract surgery for high myopia. F Cataract Refract Surg 1995;21:35-8.

5 Modarres M, Pazrvaresh MM, Hashemi M, et al. Inadvertent globe perforation during retrobulbar injection in high myopes. Int Ophthalmol 1997-8;21:179-85.

6 Totan Y, Bayramlar H, Cekic O, et al. Bilateral cataract surgery in adult and pediatric patients in a single session. $f$ Cataract Refract Surg 2000;26:1008-11.

7 Ramsay J, Diaper CJ, Saba SN, et al. Simultaneous bilateral cataract extraction. $\mathcal{F}$ Cataract Refract Surg 1999;25:75362 .

8 Beatty S, Aggarwal RK, David DB, et al. Simultaneous bilateral cataract extraction in the UK. $B r f$ Ophthalmol 1995;79:1111-14.

9 Muller-Jensen K, Fischer P, Siepe U. Sutureless corneal cataract surgery. Limbal release incisions for correcting astigmatism. Ophthalmology 1999;96:432-6.

10 Budak K, Friedman NJ, Koch DD. Limbal relaxing incisions with cataract surgery. I Cataract Refract Surg 1998;24:503-8.

11 Galand A, van Cawenberge F, Moosavi J. Posterior capsulorhexis in adult eyes with clear capsules. $\mathcal{F}$ Cataract Refract Surg 1996;22:458-61.

12 Nishi O, Nishi K, Sakanishi K. Inhibition of migrating lens epithelial cells at the capsular bend created by the optic edge of a posterior chamber intraocular lens. Ophthalmic Surg Lasers 1998;29:587-94.

13 Nishi O, Nishi K, Wickstrom K. Preventing lens epithelial cell migration using intraocular lenses with sharp rectangular edges. 7 Cataract Refract Surg 2000;26:1543-9.

14 Peng Q, Visessook N, Apple DJ, et al. Surgical prevention of posterior capsule opacification. Part 3: Intraocular lens optic barrier effect as a second line of defence. 7 Cataract Refract Surg 2000;26:198-213. 
Sunderland Eye Infirmary, Sunderland, UK

D Allen

consultant ophthalmologist

David Allen

Before embarking on surgery surgeons are required to obtain informed consent from the patient. In this case there are particular potential problems associated with cataract surgery caused by the "high myopia." Before discussing the option of surgery and giving appropriate information regarding risk of complications I would require a retinal opinion-in particular an examination of the peripheral retina for any signs of particular potential problems with retinal breaks. Also information about the presence of a posterior vitreous detachment would be of value - though the presence of a "posterior subcapsular plaque" in the crystalline lens means this information may need to be obtained by ultrasound examination. While it is now recognised ${ }^{1}$ that the risks of retinal detachment after extracapsular surgery in high myopes is less than with previous techniques, the presence of a complete posterior vitreous detachment reduces the chances of postoperative retinal detachment even further. ${ }^{2}$ In light of the recently published report ${ }^{3}$ that cataract occurring in middle age is associated with reduced life expectancy, he should perhaps be screened for coincidental systemic disease or risk factors.

IOL power calculation is problematic in high ammetropes. ${ }^{4}$ One of the (to date relatively unrecognised) reasons for this is the fact that a proportion of short and long eyes have a "normal" sized anterior segment. Standard IOL formulas assume that a short eye has a short anterior segment and, similarly, that long eyes have a long anterior segment, and use this assumption when calculating the effective lens plane of the IOL. The fact that a significant proportion of ammetropic eyes do not fit with this assumption is the reason for many postoperative refractive "surprises." I would therefore use the Holladay 2 formula contained within the Holladay Consultant computer program to determine the IOL power. ${ }^{5}$ This requires the entry of white to white corneal diameter as a surrogate for "anterior segment size" into the calculations. If this formula is not available then Holladay 1 or SRK(T) would be the formula of choice. ${ }^{6}$

Choice of IOL is also important for this patient. A relatively young patient tends to have a relatively large pupil and therefore needs to have an IOL optic at least $6.0 \mathrm{~mm}$ diameter in order to avoid edge effects. Should the patient later have retinal problems, the vitreoretinal surgeon will also be grateful that a larger optic has been used. A high myope has an increased possibility of complicated retinal problems after cataract surgery, and may require postvitrectomy silicone oil. Major visual problems result from adhesion of silicone oil droplets on the surface of a silicone IOL. ${ }^{7}$ Therefore, a silicone IOL would be contraindicated if posterior vitreous detachment has not occurred at the time of cataract surgery, or if there is significant retinal pathology predisposing to detachment. Silicone also adheres to other IOL materials, though to a much smaller degree. Of those materials tested to date at the Storm Institute for IOL Research (Apple DJ, personal communication, 2001) the material with the lowest silicone adhesion is the hydrophilic "acrylic" used in the Centreflex IOL (Rayner). Posterior capsule opacification (PCO) is particularly to be avoided in a high myope, as YAG laser capsulotomy (particularly if carried out early) probably increases the chances of retinal detachment. Detailed discussion of the role and importance of IOL material, IOL design, and surgical technique in the prevention of PCO is beyond the scope of this commentary. ${ }^{8}$ Suffice to say that for this patient a nonsilicone IOL with an optic at least $6 \mathrm{~mm}$, and with a track record of low PCO is required.

As a general rule, topical anaesthesia is my anaesthetic method of choice for cataract surgery. The patient experience, however, is more important to me than undertaking all cataract surgery under topical anaesthesia. Therefore I do not use topical anaesthesia regardless of the circumstances, and the nursing staff in our cataract unit make a judgment at the pre-assessment clinic of other factors which may make an injectional anaesthetic preferable for the patient. One factor taken into account is photophobia and undue sensitivity during A-scan biometry. I (and my anaesthetist colleague) would take as our starting point topical anaesthesia as the method of choice in a patient with a $>30 \mathrm{~mm}$ eye. I would schedule the patient for an operating list where I have an anaesthetist, and the patient's primary nurse and the anaesthetist together would assess whether he would be likely to tolerate surgery under topical anaesthesia with the addition of intravenous sedation. In that event I would start the surgery with the microscope light fairly low, and gradually increase the intensity to a level at which the capsulorhexis can be performed. In a patient with a fairly early cataract there will be a good red reflex, and the level of illumination required may be quite low. After the initial paracentesis incision I would inject intracameral (1\%) lignocaine. This additional anaesthetic in some way often improves light tolerance. If at this stage, and before the main corneal incision, it is clear he cannot tolerate topical anaesthesia, then I would stop, and convert to injectional anaesthesia.

The surgery would be through a temporal corneal incision, using my standard phaco technique, but with some minor modifications. 
The description of the cataract is of "posterior subcapsular plaque." This may in fact be referring to a posterior polar cataract, and if I thought that may be a possibility from my own examination of the patient I would take particular precautions. A posterior polar cataract is often associated with deficient (or even absent) posterior capsule immediately under the plaque. ${ }^{9}$ Under these circumstances, cortical cleaving hydrodissection is contraindicated because of the possibility of "blowing" the capsule. I would, however, carry out good hydrodelineation to ensure easy nucleus rotation within the epinuclear shell, and perhaps try to separate cortex from epinucleus.

As with a standard case, it is important to fashion a CCC that is centred, and that is about $1 \mathrm{~mm}$ smaller than the IOL optic. It is important that the whole circumference of the anterior capsule edge is inside the optic edge. I would lower the standard bottle height because of the possibility of overinflating the anterior chamber in a high myope. I would tend to use a lower flow and vacuum setting because of the reduced BSS inflow potential caused by the lower bottle. After removing nucleus and epinucleus I would viscodissect the cortex from the periphery towards the centre of the posterior capsule, leaving the posterior plaque undisturbed. Irrigation and aspiration of the peripheral cortex would be followed by slow careful lifting of the central posterior plaque. If in fact this is just a "standard" posterior subcapsular cataract, then removal often reveals a central fibrous plaque that cannot be removed by standard I and A. ${ }^{10}$ In that event, I would re-inject viscoelastic. It is often possible to raise an edge of this type of plaque with the cannula. If not, I would use the capsulorhexis forceps (Corydon forceps are particularly good for this) to try to raise an edge and then gently peel the plaque from the capsule. If this proves impossible it is important to assess the likely impact of such a membrane on vision. If it seems likely to cause visual problems, then carrying out a careful posterior capsulorhexis (with reservation of vitreous face) is probably preferable to YAG laser disruption in the early postoperative period. ${ }^{11}$

Finally, if there had been problems adequately visualising the peripheral retina preoperatively then in the postoperative period I would ask a retinal colleague to check the retina for any predisposing signs that may require prophylactic treatment or monitoring.

1 Alldredge CD, Elkins B, Alldredge C. Retinal detachment following phacoemulsification in highly myopic cataract patients. F Cataract Refract Surg 1998;24:777-80.

2 McDonnell PJ, Patel A, Green WR. Comparison of intracapsular and extracapsular cataract surgery, histopathologic study of eyes obtained post-mortem. Ophthalmology 1985;92:1208-23.

3 McKibbin M, Mohammed M, James TE, et al. Short-term mortality among middle-aged cataract surgery patients. Eye 2001;15:209-12.

4 Zaldivar R, Shultz MC, Davidorf JM, et al. Intraocular lens power calculations in patients with extreme myopia. $\mathcal{f}$ Cataract Refract Surg 2000;26:668-74.

5 Hoffer KJ. Clinical results using the Holladay 2 intraocular lens power formula. $\mathcal{F}$ Cataract Refract Surg 2000;26:12337.

6 Royal College of Ophthalmologists. Guidelines for cataract surgery. London: RCO, March 2001.

7 Apple DJ, Isaacs RT, Kent DG, et al. Silicone oil adhesion to intraocular lenses: an experimental study comparing intraocular lenses: an experimental study comparing
various biomaterials. 7 Cataract Refract Surg 1997;23:53644.

8 Apple DJ, Peng Q, Visessook N, et al. Eradication of posterior capsule opacification: documentation of a marked decrease in Nd:YAG laser posterior capsulotomy rates noted in an analysis of 5416 pseudophakic human eyes obtained postmortem. Ophthalmology 2001;108:505-18

9 Osher RH, Yu BC, Koch DD. Posterior polar cataracts: a predisposition to intraoperative posterior capsular rupture. f Cataract Refract Surg 1990;16:157-62.

10 Vasavada AR, Chauhan H, Shah G. Incidence of posterior capsular plaque in cataract surgery. J Cataract Refract Surg 1997;23:798-802.

11 Galand A, van Cauwenberge F, Moosavi J. Posterior capsulorhexis in adult eyes with intact and clear capsules. $\mathcal{F}$ Cataract Refract Surg 1996;22:458-61. 


\section{Overview}

Two leaders in cataract surgery provide a superb overview of the problems of cataract surgery in a youngish myopic male. I am pleased that they both suggest a retinal opinion before the surgery. In fact, this particular patient did have a peripheral round break and was referred to a vitreoretinal surgeon and had laser treatment. Informed consent is very much in the news with recent publications from the British Medical Association ${ }^{1}$ and the Department of Health, and it is imperative that patients are warned of the risks of surgery. The recent guidelines for cataract surgery from the Royal College of Ophthalmologists ${ }^{2}$ provide excellent information on the risks and benefits for all patients undergoing cataract surgery.

There are two problems with regard to the IOL choice-first, the choice of power. Richard Packard quotes $69 \%$ of highly myopic patients within 1 dioptre of desired refractive outcome, but what of the other $30 \%$ ? Myopes always wish to remain myopic and I would certainly agree with him in aiming for at least $0.75 \mathrm{D}$ of myopia. Assuming the biometry is $1 \mathrm{D}$ out, the patient will at worst be emmetropic and most myopes could cope well with a -2.00 postoperative refraction. The second problem is to do with the choice of intraocular lens as both experts state a sharp edge appears to prevent posterior capsule opacification, which should be prevented at all costs in a myopic male. A recent paper in the Fournal of Cataract Surgery ${ }^{3}$ stated that "being a man, being 60 years or younger and especially having an axial length of $24 \mathrm{~mm}$ or greater are associated with detachment following capsulotomy." There is a difficult choice between possible edge effects and glare from the high refractive index of an Acrysof lens versus the low posterior capsule opacification rate. Farbowitz and Davison in separate papers have recently documented visual complaints and dysphotopsia with the Acrysof intraocular lens. ${ }^{45}$ The patients who seemed to have the most glare and edge effect are, in general, patients aged under 60 who were implanted with a $5.5 \mathrm{~mm}$ optic lens. The newer designs of the Acrysof (Alcon) are one piece with an opaque sharp edge reducing the edge effect. In a patient of this age I would certainly suggest a $6 \mathrm{~mm}$ optic intraocular lens with a sharp opaque edge, either an Acrysof or a Centerflex IOL (Rayner) as David Allen suggests. A multifocal IOL would obviously be of great benefit to this patient who would like good unaided distance vision. As a myope he is used to reasonably good unaided near vision. I would not be in favour of a multifocal intraocular lens in this particular case for two reasons. The first is that there is approximately a $30 \%$ chance that the biometry may be 1 dioptre inaccurate. A myope rendered hypermetropic even if he has reasonable near vision due to his multifocal lens will, I believe, always be unhappy. Secondly, there is no multifocal IOL with a very low PCO rate-which is essential here-currently available (although they are in clinical trials).

Anaesthesia in a youngish patient with quite a lot of blepharospasm is a problem and it is interesting that each expert has a different approach. I am pleased that David Allen has highlighted that the "patient experience" is most important and I frequently use the approach of turning up the light gently and using intravenous sedation with midazolam, which is administered by an anaesthetist. This improves both the patient's and the surgeon's experience. If the patient could not tolerate a topical or subconjunctival anaesthetic which is what I use, I would then prefer to give a sub-Tenon's block on the table which would only take a few extra minutes and should take effect immediately. The use of a light general anaesthetic using benoxinate is important as we have almost forgotten the great improvements in general anaesthesia in cataract surgery, over the past 10 years.

Simultaneous bilateral surgery remains a hot topic both in the United Kingdom and abroad. There are two reasons why I would not wish to proceed with simultaneous bilateral surgerythe first is the fear of endophthalmitis and the second is the worry about the accuracy of the biometry. Despite all our endeavours to prevent endophthalmitis it still remains a problem with, anecdotally, a recent increase and I would not be happy to perform simultaneous cataract surgery until such time as the rate of endophthalmitis is well below its present $0.1 \%$. The refractive result following the first eye will give information as to the accuracy of the biometry. It will also allow the patient to realise what life is like as a low myope and it is easier then for the patient to make an informed decision as to the refractive result he would like following second eye surgery.

The two experts highlight the problems of a very deep anterior chamber when operating on a high myope. I would certainly agree that it is gratifying when the nucleus prolapses into the anterior chamber thereby facilitating its removal. I find remaining in aspiration during most of the surgery (foot position 2) prevents the trampolining effect which can be seen in the anterior chamber on moving from infusion to infusion plus aspiration. Both experts have highlighted the dangers of a plaque on the posterior capsule coming away and tearing the posterior capsule. I am not keen on a planned posterior capsulorhexis in a high myope even if the vitreous face is left intact in view of the unknown but almost certain increased risk of cystoid macular oedema. 
Both experts show how important planning is to the performance of good cataract surgery. Cataract surgery does not start in the operating theatre but rather at the consultation with the patient. In this day and age when we are told that anybody can do a cataract operation, it behoves us to look at the expertise required preoperatively, interoperatively, and postoperatively in managing what at first seems rather a mundane clinical problem.
1 British Medical Association. Report of the consent working party: incorporating consent tool kit. London: BMA, March 2001

2 Royal College of Ophthalmologists. Guidelines for cataract surgery. London: RCO, March 2001.

3 Olsen G, Olson R. Update on a long term, prospective study of capsulotomy and retinal detachment rates after cataract surgery. 7 Cataract Refract Surg 2000;26:1017-21.

4 Farbowitz MA, Zabriskie NA, Crandall AS. Visual complaints associated with the Acrysof acrylic intraocular lens. f Cataract Refract Surg 2000;26:1339-45.

5 Davison JA. Positive and negative dysphotopsia in patients with acrylic intraocular lenses. I Cataract Refract Surg 2000;26:1346-55. 